\title{
ALAALANG PAMPANITIKAN:
}

\section{Si E. San Juan, Jr. sa Panitikang Filipino}

\author{
Efren R. Abueg \\ Polytechnic University of the Philippines \\ efrenabueg@yahoo.com
}

\begin{abstract}
In an essay that he calls literary memory, the acclaimed writer in Filipino Efren Abueg provides a critical biography of E. San Juan, Jr. It begins with San Juan's coming of age at Jose Abad Santos where he became the editor-in-chief of the school paper, and explores the print culture of the period along the way. Then it transitions into the college years of San Juan in the age of decolonization at the University of the Philippines-Diliman, during which he came under the tutelage of Elmer Ordoñez, Francisco Arcellana, and NVM Gonzales, among others. Abueg, in particular, pays attention to San Juan's collaboration with Tagalog writers, emphasizing the tension between English and the vernacular and the ways in which San Juan tried to address this divide. Abueg also emphasizes the formative encounter between San Juan and Amado V. Hernandez, which hastened the radicalization of the younger writer. The essay concludes with San Juan's contribution to the development and promotion of Filipino writing, one that constitutes, according to Abueg, a critique of the continuing dominance of Filipino writing in English.
\end{abstract}

\section{Keyword}

E. San Juan, Jr., literary memory, Philippine literature, Tagalog writers, Filipino

\section{About the Author}

Efren Abueg started teaching in 1964 at MLQ University where he graduated and started his career in creative writing. In 1969, he became a part-time faculty member of the Philippine College of Commerce (now PUP) where he was active in advocating for Pilipino (now Filipino) as our national language and as medium of instruction until the declaration of martial law in 1972. He has also taught at the University of the Philippines (1974), Pamantasan ng Lungsod ng Maynila (1975-76) and later, Ateneo de Manila University (1977-78). He did consultation work with projects at Philippine Normal University and National Manpower Council (now Tesda). In 1979, he was with the Department of Pilipino at De La Salle University-Manila where he held academic posts until his retirement in 2002. For the next five years, he was writer-in-residence with the Cavite Studies Center at De La Salle University-Dasmariñas. A Ph.D. holder in translation studies from U.P., he is now mentoring students at the graduate schools of University of Perpetual Help System Dalta-Las Piňas (2007-present) and Polytechnic University of the Philippines (2007-present). A prize-winning writer in Filipino, he was given lifetime- 
achievement awards in literature (Alab ng Haraya, 2002) by the National Commission on Culture and the Arts, as well as by the governments of Quezon City (Gantimpalang Quezon sa Panitikan, 1993), Cavite Province (Gawad Emilio Aguinaldo, 2006), and the Komisyon ng Wikang Filipino (Gawad Sagisag-Quezon, 2010). 
Magkasabay kaming nag-aral ng haiskul ni Epifanio "Sonny" San Juan, Jr. sa kalagitnaan ng dekada singkuwenta (1951-1954), mga paaralang publiko na ipinagmamalaking pangunahin noon sa Pilipinas. Siya, taga-Maynila, sa Jose Abad Santos (Manila South) High School sa Meisic, Reina Regente, malapit sa Divisoria; ako, taga-Cavite, sa Arellano (Manila North) High School, halos nasa dulo, patimog ng Teodora Alonzo, Sta. Cruz, kalyeng tumutuhog patawid sa Azcarraga tungo sa Aranque Market na nakatalikod naman sa kalye Ongpin. Ipinangalan ang unang pampublikong mga haiskul sa Maynila sa mga mahistrado ng Korte Suprema ng kolonyal na administrasyon ng mga Amerikano sa Pilipinas: Florentino Torres (1844-1927), Victorino Mapa (1855-1927), Manuel Araullo (1853-1924), Cayetano Arellano (1847-1920) at Jose Abad Santos (1886-1942). Mahigit dalawang kilometro ang layo sa isa't isa ng aming pinapasukang mga paaralan at kahit nagkakatagpo ang mga estudyante ng dalawang haiskul, hindi kami nagkakilala noon ni Sonny.

\section{NANGANGAKONG BUKAL NG INISYATIBA, SIPAG, AT DEDIKASYON}

Naiiba ang pambayang karakter ng mahistradong ipinangalan sa haiskul na pinasukan ni Sonny-si Jose Abad Santos. Naglingkod sa rehimeng kolonyal ng mga Amerikano, malaking pagpapahalaga sa inaasam na pagsasarili ng sariling bayan ang ginawang pagpapakasakit nito. Hinirang na pansamantalang pangulo ng Pilipinas si Abad Santos (Marso 17, 1942) nang "itakas" ng seguridad ng Estados Unidos ang Pangulong si Manuel Quezon patungong Amerika via Australia. Sumumpang pangangalagaan ang tungkuling napaatang sa balikat nito, iniwasan ni Abad Santos ang dragnet ng hukbong militar ng Japan. Gayunman, noong Mayo 7, 1942, natutop ito ng mga Hapones sa Malabang, Lanao, kasama ang anak na si Jose, Jr. Kahit alam ang implikasyon ng pagkadakip dito, mahigpit nitong tinanggihan ang alok na makiisa sa mga Hapones sa pagpapasuko sa nakikipaglabang kawal at gerilyang mga Pilipino. Kaya nang patawan ng parusang kamatayan, nag-iwan si Abad Santos ng ganitong mga kataga sa nalulunos niyang anak: Huwag kang umiyak, Pepito...ipakita mo sa mga taong ito na matapang ka. Isang karangalan ang mamatay alang-alang sa bayan mo. Hindi binibigyan ng ganitong pagkakataon ang lahat."

May ibabaon sa kamalayan ng mga estudyanteng tulad ni Sonny ang pagpapasiya ni Abad Santos-matigas na batong hindi paaagnas sa kabilaang rahuyo at dahas na alok ng puwersa ng "bagong" imperyalista. Sa pagbubukangliwayway ng kaisipan ng mga kabataang sumasaksi pa lamang sa mga dula ng kontrakdiksiyon sa mga lipunan ng mundo, malaking pagdiriwang para sa mga ito ang makabasa ng naratibo ng gayong kadakilaan. Nakapupukaw iyon sa isip at kalooban ng sino mang humahawak ng mahalagang tungkulin sa maagang gulang, halimbawa'y tulad ni Sonny na editor-in-chief ng kanilang opisyal na pahayagang panghaiskul, 
ang Jose Abad Santos Gazette. Magiging simbolo iyon ng pagluwalhati nino mang kabataan sa panimulang mga bukal ng halagahan ng inisyatiba, sipag at dedikasyon nito sa pag-aaral.

May hiwatig na ng kaligiran ng edukasyon ang landas na tatahakin ni Sonny. Gradwado sa Unibersidad ng Pilipinas sa Padre Faura bago nagkadigma ang mga magulang niyang guro sa haiskul at sa kolehiyo. May kopya na sa kanilang bahay ng mga sinulat nina Jose Garcia Villa (Footnote to Youth) at Salvador P. Lopez (Literature and Society), gayundin ng antolohiya ng mga sanaysay na pinamatnugutan ni Vicente M. Hilario, saka mga teksbuk sa wika at panitikan. Nababasa at kilala ng pamilya nila si Manuel Viray, makata at kritiko sa Ingles na unang nagpayo sa kaniya na magsulat. Nabasa na niya sa teksbuk ng Wikang Pambansa (WP) ang tulang "Aklasan" ni Amado V. Hernandez. Masugid na rin siya sa pagbabasa ng Literary Apprentice at Philippine Collegian na iniuuwi ng kaniyang mga magulang.

Kapwa naakit kami ng opisyal na mga pahayagang pampaaralan: si Sonny, editor-in-chief ng Jose Abad Santos Gazette; ako, patnugot sa balita ng pahayagan sa Wikang Pambansa ng Arellano High School, Ang Tambuli.

Nililimbag noon sa imprenta ng diyaryong Bagong Buhay (naging Taliba pagkaraan) sa kalye Lepanto (katabi ng University of the East) hindi lamang ang pahayagang pang-estudyante ng Jose Abad Santos High School kundi ang iba pang pahayagan ng pampublikong mga haiskul na tulad ng Torres, Arellano, Mapa at Araullo. Sa "pagsasara" ng mga pahina sa press work, karaniwang nagkakasama sa imprenta ang mga kagawad ng patnugutan ng mga pahayagang pampaaralang nabanggit. Kabataang ibig ihantad sa kapwa estudyante ang kanilang mga nabasa at natutuhan, masigla ang kanilang mga diskusyon at pagtatalo roon na nagkakaroon ng ekstensiyon sa taunang/panahunang mga kumperensiya, seminar at workshop sa journalism, panitikan at malikhaing pagsulat. Malaking bentahe para sa mga kabataang ito ang pamamatnugot at pagiging staff member sa pagpapatuloy ng pag-aaral nila sa mga kolehiyo o unibersidad. Hindi lamang sila nagkakaroon ng matibay na tiwala sa sarili; nangangako pa ang kanilang mga posisyon ng scholarship (libreng tuition fee, iba pang gastusin) at ng popularidad sa kampus ng papasukan nilang kolehiyo/unibersidad.

May isang dahilan kung bakit hindi kami nagkatagpo ni Sonny noong haiskul. Kahit may mga kumperensiyang idinaraos ang Public Secondary Schools Press Association (natatag, 1931), tanging mga manunulat sa Ingles ang ipinadadala ng aming paaralan (maaaring gayon din sa iba) sa taunang kumperensiyang iyon. Nasa pangatlong taon na ako (1952-1954) nang tuluyan akong maging abala sa Ang Tambuli, pahayagan namin sa Wikang Pambansa (WP). Tumutula at nagkukuwento na rin ako, ngunit ibig kong magsulat din sa Ingles dahil binabasa 
ko ang mga katha ni Mauro Flores, hinahangaan kong manunulat at literary editor ng The Chronicler, pahayagan naman sa Ingles ng Arellano High School. Kakilala na pala noon si Sonny ni Mauro Flores na nakakasama ko sa pangkalahatang mga miting. Gayunman, sinikil ko ang hangad kong iyon dahil sinabihan ako ng kapwa ko kagawad ng patnugutan na ako'y magiging “taksil sa wika” kapag nagsulat ako sa Ingles-ganoong kakitid ang pananaw naming mga mag-aaral noon. Presidente pa ako ng Kapulungan ng Inang Wika (KIW), ang samahan namin sa WP na "simbolo" ng aming pagkamakabayan. Sintomas ang kaisipang iyon ng parang dagat na pagkakalayo ng "bakyang" panitikan sa Tagalog at sa iba pang katutubong mga wika sa Pilipinas sa "class" o mataas na uring panulatan sa wikang Ingles, huwad na paniniwalang ibinunga ng imposisyon sa bansa ng kolonyalismong Kastila at ng "demokrasya" na hatid ng imperyalismong Amerikano. Panahon din iyon ni Presidente Ramon Magsaysay, ang "American boy" na sumugpo sa rebelyon ng mga Huk (dating Hukbong Bayan Laban sa mga Hapones/Hukbalahap) at lalong nakapagpahina ng pakikipaglaban para sa mga karapatan ng mga unyonista, mga magsasaka sa Gitnang Luzon at mga anakpawis sa mga tubuhan sa Tarlac, Negros, at iba pang mga lugar ng asukarera sa Pilipinas.

Sa pag-aaral namin ni Sonny sa halos magkatulad na kapaligiran (Binondo, San Nicolas, Tundo, Sampaloc at Sta. Cruz) ilang taon pagkaraan ng digma, ang kamalayan ng pagkakaiba sa pananamit, itsura at pagdadala ng sarili at personal na mga gamit ang maaga rin na nagpamalay sa kabataang tulad namin ng maluwang nang agwat ng kalagayan noon sa lipunang Pilipino. Sabi ni Sonny: Halos $80 \%$ ng mga kaklase ko sa Abad Santos ay taga-Tundo at karatig; marami ang nagtatrabaho sa San Nicolas at Dibisoria. Noong nasa elementari ako (Andres Bonifacio Elementary School sa Tayuman), meron pang nakapaang mga estudyante noon. Marungis at marumi ang mga kalaro ko...

Kung may pribadong elitistang mga paaralan noon, may publikong mga paaralan naman para sa mga uring nasa gitna (petiburges) at nasa ibaba (masa/ dukha). Kadlo ng kaayusang itinindig ng mga Amerikano ang uring nasa gitna, pataas, yaong mga ilustrado na pagkaraang tulungang masugpo ang "insureksiyon" ng mga kababayan pagsampa ng ikaladalawampung siglo (1900) ay nagsihawak ng mataas na mga tungkulin sa gobyernong malaon ay tinawag na Komonwelt, modelong sunod sa imperyalistang kaayusan ng Great Britain. Kailangan noon ang matinding pagsisikap at pagkitil sa pagkasungyaw ng mga dayukdok na nasa ibaba para makaakyat sa gitnang uri. Gayon ang historikal na proseso ng pag-angat sa kabuhayan, oportunidad at sakali'y prestihiyo para sa ordinaryong pamilyang Pilipino, kasama na rito ang mga kapatid na minoryang etnikong grupo.

Hindi kami nagkalayo ni Sonny ng mga kapaligirang pinaglinangan ng aming kabataan bago namin naunawaan ang implikasyon ng pagkakaroon ng hiyarkiya 
ng lipunan sa Pilipinas. Kung pauwi siya mula sa Jose Abad Santos High School (kanto ng mga kalye Meisic at Reina Regente sa Binondo) babaybay siya pahilaga sa mahabang kalyeng Jose Abad Santos, kakanan pagdating ng Tayuman na kinaroroonan naman ng pinagtapusan niyang Bonifacio Elementary School at pasilangang tutumbukin ang kalye Oriqueta, saka magpapahilaga sa mga kalye Tayabas, Batangas, Laguna at Antipolo na kalapit naman ng Blumentritt. Kapareho rin siguro ang ruta niya sa kaniyang pagpasok sa iskuwela: mula sa tinitirhan, maaari naman siyang maglakad pakanluran hanggang dulo ng Blumentritt, hahakdaw sa kalye Antipolo at lilikaw sa dulo ng mga kalye sa kaliwa ng Abad Santos hanggang sa makarating siya sa Reina Regente. Ang mga lugar na ito noong nasa haiskul kami ay larawan ng karalitaan at kawalan ng kaayusan maliban sa ilang negosyong kumikita na dahil hindi pa nagkakarami.

Naglalakad din ako tatlong araw sa isang linggo mula sa Arellano High School sa kalye Teodora Alonso, Sta. Cruz para makapagtabi ng 30 sentimos na pambili ng isang kopya ng magasing Liwayway tuwing Biyernes (nasa elementarya pa lamang ako, ipinag-uuwi na ako ng kopya nito ng aking ina tuwing namamalengke). Kasama ang may sampu o higit pang mga kaklase na pauwi sa Norte ng Maynila, (papuntang Manuguit sa halos dulo ng Avenida Rizal at katapat ng Chinese Cemetary), magsisimula kaming maglakad ng mga kaklase ko pahilaga mula kalye T. Alonso, kakanan sa Lope de Vega, kakaliwa at babaybayin ang Avenida Rizal hanggang sa Antipolo, Cavite, Tecson, at ako na lamang ang estudyanteng naglalakad paglampas ko sa Manuguit, at iisa-isahin ko nang lampasan ang mga kalye sa Grace Park, mula $1^{\text {st }}$ Avenue hanggang makaabot sa kanang bahagi ng $7^{\text {th }}$ Avenue, saka kakanan ako sa $7^{\text {th }}$ St. na tatlong bloke pa ang hahakbangin bago ko marating ang bungalow ng aking tiya (pinsan maikatlo ng aking ina). Gasgas ang mga takong ng aking sapatos sa gayong paglalakad, nasusubok ang tatag at tapang ko sa pagharap sa walang katiyakang buhay. Ganoon din marahil ang pakikitunggali para sa kabuhayan ng mga pamilya ng kabataang mga estudyante na kasabayin namin ni Sonny sa panahong mabagal, ngunit patuloy namang bumabangon sa nakaraang digma ang Maynila, pati na ang mga negosyong matumal bunga ng kakulangan ng produksiyon at mga pangunahing pangangailangan sa mga pamilihan para sa mga dukha. Sabi nga ng senior naming mga kaklase-mabuti pa ang Chinese cemetery, "laging may mga pagkaing kulay ginto sa mga altar nito."

Ilang ulit akong nakarating sa Abad Santos High School, kabuntot ng mga kaklase at kabarkadang dumarayo sa pagbabasketbol doon, at kung nagkakaroon ng "hindi pagkakaintindihan" ang mga koponang naglalaro, at magkaroon ng kaunting "gulo," sisibad na ako ng takbo pasilangan sa Soler at papasok sa Aranque Market sa T. Alonso, saka tatawid sa Azcarraga (Recto ngayon) at babalik na sa gusali ng Arellano High School. Sa akin, malayo na sa Arellano ang simbahan ng Binondo sa Plaza Calderon dela Barca (Plaza San Lorenzo Ruiz ngayon). Narating ko lamang 
ang Escolta nang magtrabaho na ako noong 1958 sa kalye Juan Luna, malapit sa ilog-Pasig at doon ko rin unang natuklasan ang mga iskuwater na naninirahan sa mga guho ng digma, ilang gusaling lugmok na at hindi pa naisasama ng pamahalaan sa programang rehabilitasyon.

\section{PAGHUBOG SA KAMALAYAN, PAGPAPATALAS NG PANULAT}

Noong 1954, pumasok si Sonny bilang iskolar sa Unibersidad ng Pilipinas (U.P.). Isang pagmamalaki ng kabataang tulad niya ang makapag-aral sa isang unibersidad na may malawak na reputasyon hindi lamang bilang pangunahin sa propesyonal na mga kurso at pangangalaga sa moral at intelektuwal na kagalingan ng kabataan kundi pati na ng "kalayaang pang-akademiko" para sa buong komunidad. Dahil sa huwarang imahe ng U.P., hindi agad nasakyan ni Sonny ang pampaibabaw na mga pangyayaring pang-araw araw sa kampus. Hindi pa nakasilid sa kamalayan niya na may nagtutunggaliang mga diskurso ng dalawang pangkat sa unibersidad: mga estudyante at propesor na nasa anino ng simbahang Katoliko at ang mga sekular na may haplos ng iba-ibang kulay: mga liberal sa oryentasyon, free thinkers, at mga makabayan (nationalists). Mainit na ang polemikang isinusulong na noon-ang panukalang-batas ni Claro M. Recto ukol sa pagtuturo ng "buhay at mga gawa" ni Rizal sa lahat ng antas ng pag-aaral.

Naging buhay na karanasan ni Sonny ang kabiguan sa inakala niyang huwarang imahe ng U.P. nang maging baguhang reporter siya ng Philippine Collegian (PC). Hindi lamang di malalathala sa mga pahina ng PC ang ulat niya tungkol sa pagkamatay sa hazing ng isang estudyante sa kampus ng Diliman, kundi "masesensor" pa ng mismong presidente ng unibersidad ang isang tula niyang naglalaman ng salitang may apat na letra. Makukumpirma niyang bunga iyon ng pangingibabaw ng pangkating sektaryan doon na umani ng reaksiyon mula sa mga propesor na "progresibo" kabilang sina Dr. Ricardo Pascual (pilosopiya), Dr. Leopoldo Yabes (panitikan), at Teodoro Agoncillo (kasaysayan). Nabalanse ang negatibong karanasang iyon nang talikuran niya ang pagdodoktor at kunin ang Bachelor of Arts (English). Pinaunlad niya ang kaniyang pagsusulat sa haiskul sa gabay ng mga propesor niyang sina Elmer Ordoñez, Francisco Arcellana, at NVM Gonzales. Sa mga araling Comparative Literature (sa ilalim ng mga propesor na sina Cristino Jamias, Alfredo Morales, at Leopoldo Yabes) at pilosopiya (sa gabay naman nina Propesor Armando Bonifacio at Ricardo Pascual), waring pinaghanda siya sa magiging malaking "trabaho" niya sa hinaharap, ang kritisismo, mga araling pangkultura, at ideolohiya.

Sa pagsusulat, minodelo ni Sonny ang propesor niyang si Francisco Arcellana (Pambansang Alagad ng Sining, 1990) dahil sa "pagka-metikuloso" nito sa gramar 
at komposisyon sa Ingles, tulad ng pagsulat ng talata, pagbubuod ng diwa na batay sa analisis ng mahuhusay na (mga piniling) tekstong pampanitikan. Hinangaan niya ang pagiging "matiyaga, mapagpasensiya, (at) kaaya-ayang pagbibigay-payo" nito na naging kapaki-pakinabang sa kaniya. Isa sa mga bunga ng disiplina niya sa paggiya ni Prop. Arcellana ang unang tula niya sa Ingles na inilathala noong freshman siya sa Literary Folio ng Philippine Collegian.

Kinilala pa ni Sonny ang pagkadagdag sa "paghubog ng pananaw/kaisipan ng kanilang batch" hango sa "masalimuot na kapaligiran ng mga guro, mga estudyante, mga administrador, karaniwang mga empleyado, kasama na ang mga janitor at mga manggawa sa Basement Cafeteria (lugar na pinanghanguan din ng tradisyon ng pagtatalo/diskursong pang-intelektuwal), mahigpit na mga pangangailangan sa kurikulum at mga disiplinang pangkaisipan, mapagpalalim na mga teksbuk, saka mapagliming pagtuturo, malayang pag-iisip (at pagsasalita) at mapangahas na eksperimentasyon sa larangan ng pisikal at mental na ekspresyon ng aming mga pangarap at paghahangad."

\section{PAGHAKDAW SA LABAS NG PAMANTASAN}

Sa panahong nag-aaral si Sonny sa U.P. (magna cum laude, 1958), nakapangingibabaw na gabay noon sa pagsusuring pampanitikan ang mga teorya nina I.A. Richards (Principles of Literary Criticism/Practical Criticism), John Crowe Ransom (New Criticism), at T.S. Eliot (Tradition and Individual Talent/Hamlet and His Problems) na karaniwang tinatawag na formalismo. Impluwensiyado nito ang silabus ng mga kursong pampanitikan sa U.P ng mga propesor-iskolar na galing Amerika noong dekada singkuwenta/sisenta o maaga pa sa rito. Hango lamang sa teksto ang halagahin ng tula, hindi sa ano mang "nanggagaling sa labas" nito kaya pangangailangang mga babasahin (readings) sa panitikan ang mga akda ng modernistang sina Joseph Conrad, Henry James, Virginia Woolf, Gertrude Stein, pati na si T.S. Eliot (Wasteland) na paborito ng kabataang mga makata noong dekada '6o. Kaming mga estudyante ng MLQ University na pumupusturang manunulat ay "haling na haling" kina Ernest Hemingway, William Faulkner, D.H. Lawrence, F. Scott Fitzgerald at iba pa. Malaon, kasama rin sa mga diskusyon namin sa mga karinderya sa R. Hidalgo ang mga eksistensiyalistang sina Camus, Sarte, Kierkegaard at Nietzsche. Dumarami na sa panahong iyon, tulad ni Sonny, ang tumatalikod sa mga obra ng kilalang mga manunulat na iyon. Marahil, si Sonny (kami naman ay naudlot sa pag-aaral ng Asian Studies sa U.P noong dakong 1963) ay nakararating din sa Popular Bookstore sa gilid ng kampus ng Mapua Institute of Technology sa Doroteo Jose. Natuklasan ko na ang nasabing bookstore bunga ng "pagsawa" ko sa mga babasahing libreng ipinamimigay ng United States Information Services (USIS). Sa tulong ng mga empleyado roon na sina Willie Orbito at Hugo 
Agustin, nagiyahan ako sa pagbili ng mga pamplet na rekomendado nila tulad ng sinulat nina Renato Constantino, Hernando Abaya, Alejandro Lichauco, at Teodoro Agoncillo.

Nasa U.P. pa lamang, nakikihalubilo rin si Sonny sa mga peryodista't mga manunulat sa media na mga alumnus ng U.P. Sabi niya: "kaibigan ko na noon ang kilalang mga manunulat mula sa kampus na sina Rony Diaz, Adrian Cristobal, Alex Hufana, at Pic Aprieto" na nagsusulat sa Manila Times (Florentino Torres St.), Free Press (Avenida Rizal), bukod pa kay Andres Cristobal Cruz na naging kagawad ng patnugutan ng magasing Liwayway (Soler-Calero Sts.). Sa may kanto ng Avenida Rizal (at Soler) sa gawing kanan, naroon ang tanggapan ng Philippines Free Press. Pagtawid naman ng abenidang ito, tutumbok ang Soler sa kalye Florentino Torres na kinaroroonan sa gawing kaliwa (may 20 metro ang layo) ng Manila Times. Magkakalapit halos ang mga publikasyong iyan na hindi mapalalampas ni Sonny na di mapasyalan. Kung ano ang natuklasan at natutuhan niya sa mga publikasyong iyon, bahagi iyon ng pagpapalawak ng mundo ng isang estudyanteng manunulat na tulad niya.

Nakasisiya marahil sa isang bagong mananaliksik na matuklasang sa mahigit na dalawang dekada lamang, nag-ugat na ang panitikang Anglo-Amerikano sa Pilipinas nang makasulat si Paz Marquez (naging Benitez), guro sa malikhaing pagsulat sa U.P., ng unang "makabagong maikling kuwento sa Ingles" ("Dead Stars"). Naging modelo ang kathang iyon sa formalismo/ tekstuwal na dulog sa panitikang inaaral sa mga kampus. Hinangad pa ni Leopoldo Yabes ang mataas na standard ng panulatan sa Ingles sa kabila ng husay ng mga obra nina Loreto Paraz-Sulit, Paz Latorena, Arturo Rotor, Casiano Calalang at iba pa-isang patunay ng walang katapusang hangaring pantayan kundi man lampasan ang kalidad ng panitikan na sunod sa tradisyong kanluranin. Na tinugon naman ng reaksiyon ng mga manunulat noong dekada trenta sa wikang katutubo (Tagalog) na may bahid ng Marxismo-Leninismo mula sa mga manunulat ng Estados Unidos na sina Upton Sinclair, Mary Heaton Vorse, Clifford Odet, Mike Gold at iba pang tinawag na Kaliwa. Uwi ang bagong kalakarang pampanitikang ito ng "pensiyonadong" mga Pilipino na nag-aaral sa Estados Unidos tulad ni Salvador Lopez ("Literature and Society”), isa sa mga tagapagtatag ng Philippine Writers' Guild noong panahon ng Komonwelt. Nauna nang nalathala ang nobelang Banaag at Sikat ni Lope K. Santos noong 1907 na sinundan ng mga tula, awit at salaysay na nasulat bunga ng kabalisahan ng mga magbubukid at manggagawa sa industriya ng asukal at niyog sa Sentral at Katimugang Luzon. Hindi na kaila ang mga pangyayaring pampanitikang ito kay Sonny_-isang pagkilala sa rebolusyonaryong tradisyon ng panitikan ng mga Pilipino mula pa sa Panahon ng Propaganda hanggang Rebolusyong EDSA nitong 1986. Waring ang pana-panahong pagsariwa sa tradisyong iyan ay pagpapagunita ng pananaig pa rin ng maraming akdang Pilipino, hindi eksklusibo sa Ingles ng mga 
akdang tiwalag sa diwa at damdamin sa mga kontradiksiyon at tunggaliang umiiral sa lipunan. Ang maagang obserbasyong iyan ng mga manunulat ay unti-unting nagkaroon ng manipestasyon sa ilang mga akda sa mga patimpalak ng samahan ng mga pitak sa Wikang Pambansa (WP) ng mga pahayang pangkolehiyo, ang KADIPAN (Kapisanang Aklat, Diwa at Panitik) noong dekada singkuwenta-sisenta na nag-ani ng mga kathang realista/ malao'y realistang ng sosyal ng kabataang mga manunulat sa MLQ University (1964-1972). Sa kakulangan ng malalapitang mga manunulat sa Ingles (may ilang manunulat sa WP noon na nakaugat sa lupa) na gigiya sa sumisikal na mga manunulat doon, ang napagbalingan nila'y ang realidad ng maitim, palikaw-likaw, mabahong estero sa ilalim ng makitid na tulay na ginagapangan ng nagsisiksikang mga jeepney na naghihintay ng mga pasahero sa dulo ng Kalye R. Hidalgo. Masuwerte ba ang mga taga-MLQU na sina Rogelio Sicat, Dominador Mirasol, Rogelio L. Ordoñez, at Edgardo M. Reyes na minodelo ang naging mga kaibigan ni Sonny na sina Alejandro Abadilla, Amado Hernandez, at iba pa dahil hindi napalapit kina Jose Garcia Villa o Nick Joaquin?

\section{DALAWANG ENGKUWENTRO NG PAGPAPASIYA}

Habang nasa U.P., umuukilkil na rin sa isip ni Sonny ang mga tanong ukol sa direksiyon ng kaniyang pagsusulat at ang wika niyang gagamitin sa paglikha. Aaksiyunan niya ito pagkaraang "galugarin" at matuklasan siya ng Panitikang Tagalog/Pilipino/ Filipino. Hindi naman tiwalag ang mga tanong na iyan sa pagkatao niya: Tagalog ang wika ng kamag-anakan niya sa Montalban, Rizal, at iyon ang ginagamit nilang wikang pang-araw-araw sa bahay at sa kampus ng U.P. (19541958). May pitak sa WP ang Jose Abad Santos Gazette. Sa U.P naman, malakas na rin ang kilusang pangwika sa suporta ng mga estudyanteng sina Oscar Suarez-Manalo (pangulo ng Kapisanang Tagalog) at Lucila Potenciano at mga kasama sa gabay nina Propesor Tomas Aguirre at Teodoro A. Agoncillo, puno ng Departamento ng Kasaysayan. Bilang isa sa mga haligi ng wika at panitikang Tagalog, aksesibol kay Sonny ang mga risert ni Agoncillo sa panitikang Tagalog mula sa panahon ng grupong Aklatang Bayan, Ilaw at Panitik hanggang sa panahon ng Kapisanang Panitikan bago magkadigma. At aktibo na rin ang KADIPAN na nabuo noong 1950 sa mga kolehiyo at unibersidad sa antas tersiya, kalahok ang nakatatandang mga manunulat na sina Alejandro Abadilla at Amado V. Hernandez, ang huli'y mapahahalagahan niya "nang malayo" sa dalawang pagkakataon bago tuluyang masaklaw ng kaniyang pagsusuring pampanitikan.

Una, sa seremonya ng pagkakaloob kay Nick Joaquin ng Stonehill Awards (1958), sunod sa pangalan ng negosyanteng si Harry Stonehill, dating sundalong Amerikano na naging malaking negosyante sa Pilipinas pagkaraan ng Ikalawang Digmaang Pandaigdig. Nagkataong natuklasan ng nag-iimbestigang si Justice 
Secretary Jose W. Diokno na may iniingatang "black book" si Stonehill-listahan ng matataas na opisyal ng pamahalaan na umano'y "sinuhulan" nito kasama na si Presidente Diosdado Macapagal, dating presidente Carlos Garcia, at pati na ang mga lider ng partidong nasa mayorya (Liberal) at oposisyon (Nacionalista). Nang may posibilidad nang tumestigo si Stonehill laban sa mga opisyal na ito na maaaring maging basehan ng impeachment ni Presidente Macapagal, mabilis na idineport ng pangulo si Stonehill na ganap na "sumira" sa programang antikorapsiyon ng pamahalaan noon. Pansamantalang nasa laya noon si Hernandez at naghihintay ng resulta ng apela sa Korte Suprema sa kasong "una" sa kasaysayan ng jurisprudencia ng Pilipinas: sakdal na rebelyon, kakabit ang iba pang mga krimeng ipinagdusa na nito ng pagkabilanggo nang may anim na taon. Awtor ng Bayang Malaya (mga tula), naging alamat ang pag-akda nito sa loob ng Muntinglupa (pati na sa bartolina nito) ng mahahabang tulang natipon sa isang antolohiya, ang Isang Dipang Langit, gayundin ng ilang bahagi ng mga nobelang Luha ng Buwaya at Mga Ibong Mandaragit.

Ikalawa, nang naabsuwelto ito sa kaso (1966) at naging "celebrity" sa progresibong mga grupo. Naanyayahang magsalita sa mga fora at kumperensiya, nagturo ito sa U.P. at pati na sa Ateneo de Manila University. Sa harap ng mga manunulat ng U.P. Writers' Club, narinig ni Sonny ang hamon ni Hernandez sa "madlang natulala kabilang ang mga petiburgesyang intelektuwal sa Kamaynilaan"-bakit sinusuob ang mga manunulat sa Ingles at ipinangangalandakan ang konkistador na mentalidad (ng) tulad ni Nick Joaquin at mga alipores niya habang ang mga manlilikha sa bernakular ay sukdulang napapabayaan?" May malalim na impresyong iiwan kay Sonny si Ka Amado na mula noon ay binigyan niya ng atensiyon sa kaniyang pagbabasa at pag-aaral. Gayunman, hindi nakasagabal ang mga iyon sa pakikipag-ugnayan ni Sonny sa mga kaibigan at interesanteng mga personalidad sa kaniyang bayan habang siya'y nasa Harvard University (1962-1965). Tulad ng mga propagandistang nanatili sa Madrid at Barcelona noong panahon ng Propaganda, nakasubaybay si Sonny sa mga pangyayari at mga personalidad sa bayang tinubuan, kabilang na si Amado V. Hernandez na nabilanggo (1951) sa kainitan ng paggamit ng Kanluran sa doktrinang McCarthy (senador ng Estados Unidos) laban sa lahat ng makakaliwa't progresibong puwersa't kilusan noong Cold War.

\section{PAGKAUNAWA SA SARILI AT TUNGKULIN SA LIPUNAN}

Maaaring nagugunita pa ngayon ni Sonny ang alingawngaw ng tinig ni Hernandez sa isang talumpati nito sa isang pagtitipon sa U.P. na kumukuwestiyon kung bakit tiwalag sa ating panitikan sa mga wikang bernakular ang mga institusyon at isip at puso ng mga edukado at nakaangat na sambayanang Pilipino. Naisip din niya marahil 
ang ilang tula niya sa Pilipino na inilathala ng Philippine Collegian. At umuukilkil pa sa kaniyang kaloooban ang kuwestiyon ng pagsulat sa kaniyang Inang Wika nang isinasakonsepto na niya ang ebalwasyon sa mga akda nina Jose Rizal, Carlos Bulosan, Lope K. Santos, Jose Maria Sison, una na ang kay Amado V. Hernandez. Hindi ang kaniyang pagiging Manileño o wikang nilakhan niya kundi ang malalim na pagsusuri sa sarili "kung saan siya nagmula at ano ang kinabibilangan niyang uri" ang nagtulak sa kaniyang isiping umakda sa sariling wika nang hindi inaabandona ang banyagang wikang makapagpapalawak pa ng kaniyang kaalaman at pananaw at makapagpapatalas sa kaniyang mga argumento sa maraming penomenon ng mundo. At nang harapin na niya ang nobelang Banaag at Sikat, ang unang akdang binigyan niya ng maliming pag-aaral (kahit hindi pa niya nasusulat), naging ganap ang kaniyang desisyong gamitin na rin ang Wikang Pilipino sa kaniyang mga pagsusuri't pag-akda.

Marahil, dagdag na bunga iyon ng paglaki sa kamalayan ni Sonny ni Hernandez na pinipigipit ng kaso sa hukuman bunsod ng pagiging masugid na lider-obrero at progresibong peryodista nito. Nang nasa Harvard siya, humantong ang palitangsulat nila sa pagsasalin niya sa Ingles ng ilang tula ni Ka Amado, ang Rice Grains: Selected Poems (1966). Ang antolohiyang ito at ang sumunod na inedit ni Sonny, Introduction to Modern Pilipino Literature (1974) ang naging magkasunod niyang pagpapakilala kay AVH sa mga banyagang mambabasa ng panitikang Pilipino na salin sa wikang Ingles. Kaalinsabay ng introduksiyon ni Sonny sa panitikan ng Pilipinas sa Wikang Pilipino, nakatulong pa nang malaki sa reputasyon ni Ka Amado ang imbitasyon sa mga fora/kumperensiyang pandaigdig, tulad ng Afro-Asian Writers' Emergency Conference sa Beijing, Tsina noong Hunyo-Hulyo, 1966; International War Crimes Tribunal na inorganisa noong Nobyembre 1966 ng British logician, anti-war activist, at manunulat na si Bertrand Russel (kasama ang iba pang mga manunulat, ilan lamang sina Jean Paul Sartre at Peter Weiss) upang siyasatin ang mga patakaran at mga paglabag ng Estados Unidos sa paglulunsad ng digmaan laban sa masang mga mamamayan ng Vietnam. Bago pa nagkaroon ng OFWs, ayon sa saliksik ni Sonny, ipinahayag na ni AVH noon pang sinakop ng mga Hapones ang Pilipinas, na dapat "may iisang wika ang mga manggagawa ng mundo, higit pa sa hangganang pambansa at panlahing kapakanan...patungo sa iisang layunin (at iyan) ang paglagot sa mga tanikala ng paniniil, pasismo, at imperyalismo" (1952 Yearbook of International Longshoremen and Warehouse Men's Union, Local 37 na inedit ni Carlos Bulosan).

Sa pagkakasalin sa Ingles ng mga tula ni Hernandez, naukilkil din sa pambansang konsensiya ng Pilipinas ang "kapabayaan," partikular ng may tungkulin sa mga sektor ng edukasyon at kultura, sa paglinang sa panitikan ng Pilipinas na nasusulat sa Cebuano, Waray, Hiligaynon, Bicol, Ilocano, Kapampangan, at iba pa. Saksi si Sonny sa mga naging bunga ng pahayag ni AVH ukol sa panitikang bernakular: 
noong dekada '9o, naging masugid na ang pag-aaral sa panitikan sa iba-ibang wika ng Pilipinas, kaya nagunita ko ang inedit kong aklat, Mga Piling Akdang Pilipino (1970), koleksiyon ng salin ng ilang kuwento sa Cebuano, Hiligaynon, Ilocano, Ingles, at isang sanaysay ko tungkol sa pagkakaroon ng pambansang panitikan sa pamamagitan ng salin na binasa ko nang tanggapin akong lecturer ni Dr. Ernesto Constantino, tapangulo ng Departamento ng Pilipino, U.P. noong 1974 pagkaraang pigilin akong magturo ng martial law simula 1972.

Pag-uwi ni Sonny mula sa Harvard (1966-1967), umiinit na ang aktibismo ng militanteng mga estudyante hindi lamang sa Diliman kundi sa malalaking kampus ng mga unibersidad sa Kalakhang Maynila. Ilang taon nang hinugot mula sa mga kasapi ng Student Cultural Association-U.P. (SCAUP) ang mga iskolar na nagsipagaral ng Marxista-Leninista, na pinaniniwalaang nagluwal ng Kabataang Makabayan (KM) noong Nobyembre 30, 1964. Nang sumunod na mga taon, matatandaan ang malawakang imbitasyon ni Jose Maria Sison sa kabataang mga propesor/ guro at mga estudyante mula sa iba't ibang institusyon ng edukasyon na dumalo sa oryentasyon linggo-linggo sa Bonifacio Center sa San Francisco del Monte (hindi kalayuan sa Quezon Avenue). Kasunod pa rin ang pagpapasigla sa mga teach-in at $d g$ (discussion group) mula lungsod hanggang sa kanayunan. Bagama't nasa University of Connecticut Storrs si Sonny, nabalitaan niya ang pagkonsolida sa nagsulputang maraming organisasyong radikal na kabataan sa Pangalawang Pambansang Kongreso (Marso 15-16, 1969) ng Movement for Advancement of Nationalism (M.A.N.) sa pangunguna nina Senador Lorenzo Tañada (National Chairman) at Renato Constantino (Policy and Planning Committee Chair). Ang M.A.N. ang naging mukha ng nasyonalismo na katanggap-tanggap sa mga elitista at burges na mga Pilipino na nangamba sa paglaganap sa bansa ng Sosyalismo/ Komunismo sa mga huling taon ng dekada sisenta. Ngunit hindi nagtagumpay ang layunin ng M.A.N. dahil sa mga pagkakaibang ideyolohikal at pang-estilo sa pagsasapraktika ng mga gawain ng kasaping mga organisasyon ng kabataan tulad ng Kabataang Makabayan at Samahang Demokratiko ng Kabataan (SDK).

\section{PAGSASANAY SA WIKA, PAGPAPAHAYAG NG DIWA}

Sa pagbalik nga ni Sonny mula sa Harvard, inimbitahan siya ni Ka Amado na magsulat sa Pilipino sa peryodikong ineedit nito, Ang Masa. Lingguhan, inilalathala ito ni Serafin Lanot at inilalako ng mga aktibista. Sa kolum naman ni AVH sa arawang Taliba, mababasa ang korespondensiya ng dalawa tungkol sa mga isyung pang-internasyonal para sa kabatiran at pagdidili-dili ng masang mambabasa. Naging lalong malapit ang relasyong pampanitikan ng dalawa-isang matandang peryodista at beteranong lider-obrero at isang sumisikat na kabataang kritiko at iskolar na naglelektyur o kaya ay fellow sa mga unibersidad sa Estados Unidos, Asya 
at Europa. Noon pang Mayo 30, 1964, napawalang-kabuluhan ng Korte Suprema ang kontrobersiyal na sakdal kay AVH, desisyong naging muhon sa kasaysayan ng pag-aaral ng mga batas sa Pilipinas. Kasunod nito, nasubaybayan ni Sonny ang mga balita tungkol sa pagiging abala nito sa mga fora, sa pagsusulat sa mga magasin at peryodiko, pati na sa pagtuturo sa dalawang unibersidad, U.P. at Ateneo de Manila University. Naging lubhang malapit din dito ang militanteng mga kabataang nagpoprotesta sa mga lansangan, plasa, at saan mang espasyo maipahahayag ang mga pagtalakay nila sa mga isyu ng panahon. Kaya matatandaang nang yumao noong Marso 24, 1970, inihatid si AVH sa huling hantungan ng daan-daang mga aktibista sa mainit na pagkilos laban sa rehimeng Marcos. At dalawang taon pagkaraang yumao, ginawaran ito ng Orden ng Pambansang Alagad ng Sining sa Panitikan (Artista ng Bayan/National Artist) — na sa paningin ni Sonny at ng maraming kaisa niya sa pananaw ay pagtatangka ng rehimeng Marcos na "pagandahin" sa bayan ang pangit na imahe ng diktadurya nito. Naging Artista ng Bayan si AVH pagkaraang gawaran ng Doctor of Humanities honoris causa ng U.P. at ng Gawad Tanglaw ng Lahi ng Ateneo de Manila University.

Sa pagsusulat para sa Ang Masa, naging masigasig si Sonny sa sinasabi niyang "pagsasanay" sa paggamit sa wika ng bayan. Sa mga taong 1964-1965, nakapaglathala siya ng mga tula sa seksiyong Pilipino sa Dawn ng University of the East, inilabas din ang ilang maiikling kuwento niya sa Liwayway, bukod pa sa pagiging kontribyutor niya sa Free Press sa Wikang Pilipino. Isang taon bago suspendihin ang writ of habeas corpus, napabilang na ang isang kuwento niya sa antolohiyang Sigwa, ang nagkaisang rebolusyonaryong tinig ng mga manunulat sa isang aklat na inilathala bago ideklara ang batas-militar. Hindi pa kasama rito ang "pakikipagkaibigan" niya kay Alejandro Abadilla na humantong sa napag-usapan nilang paglalathala ng kritika sa magasing Panitikan dahil malaon, mapaninindigan ni Sonny na "kung walang kritikal na kamalayan at praktika, mahirap makupkop o mabawi muli ang isang awtentikong kamalayang historikal na saligan ng isang mapagbago't maunlad na kilusan." Noon pa man (1964), kahit okupado ang isip niya kung paano isasagawa ang tunay na pagsulat sa Pilipino, ang mahalaga'y ang wikang gagamitin niya na may perspektibang pangkasaysayan at batayang materyal dahil sa gayong paraan lamang niya maitataguyod ang sarili sa proyekto niyang iyon. Bago siya nagbalik sa mundo ng pagtuturo at pananaliksik at iba pang aspekto ng iskolarsip sa Estados Unidos, nailagay na siya sa angkop na "sityo" ng panitikan sa wikang Pilipino-isang pagkilalang hindi na kaila sa marami pang kabataang sumusulat sa Ingles na naging mga iskolar ng wika at panitikan sa wikang Pilipino, tulad nina Rose Torres-Yu, Soledad Reyes, Patricia Melendrez-Cruz, Mario Miclat, at iilan pang iba.

Sa punto ito ng pagbalik ni Sonny sa Pilipinas, gaano man kaikli ang panahong iyon, hindi na lamang wika ang niresolba niya sa sarili. May malaki nang kontrakdiksyon sa lipunang Pilipino noon at mga kabataan ang nagsusuri at 
gumagawa ng mga paraan para malutas ito. Kaya isang malaking pang-akit ang ipagpatuloy ang pakikipag-ugnayan sa mga peryodista at manunulat noong nagaaral pa siya sa U.P. Habang nagtuturo sa alma mater niya (U.P.) at sa Centro Escolar University minsan sa isang linggo, dumadalaw siya sa progresibong mga institusyon ng edukasyon, una na rito ang pinakadukhang kolehiyong pampubliko sa Pilipinas na kaibayo lamang sa kalye Lepanto ng mayamang University of the East, ang Philippine College of Commerce (PCC) na ang "tahanan" ay isang pinakalumang gusaling pamana pa ng mga Amerikano. Malaon, sa kalagayang pisikal ng kolehiyo na kabaligtaran ng mga "bago't progresibong” pananaw at kaisipan ng mga propesor at pangunahing mga estudyante nito, tinawag itong "hotbed of activism." Nakilala niya roon si Dr. Nemesio E. Presidente, pangulo ng kolehiyong ito na naging lunduan ng pagtuturo ng may iba't ibang kaisipan sa kampus nito sa University Belt bago ito naging Polytechnic University of the Philippines (PUP) sa Mabini kampus sa Sta. Mesa, Maynila. Kinikilala si Prudente hanggang sa ngayon na isa sa iilang pinakaprogresibong akademisyan ng bansa. Isinakripisyo nito ang posisyon, kaalwanan sa buhay at seguridad sa loob ng kampus nang tahasan nitong nilabanan, kasama ang mga estudyante't masa ang makapangyarihang mga aparato ng rehimeng Marcos (1965-1986). Dagdag pa noon ni Sonny: “... nakahuntahan ko sina Isabelo Crisostomo, Anacleto I. Dizon, Teodosio Lansang, Florentino Dauz, Cesar Mella, atbp." Premyadong manunulat sa Ingles at bayograper ng mga Presidente si Crisostomo, makata namang "avant-garde" sa Ingles sina Dauz at Mella; nanirahang matagal sa Russia si Lansang (Teodosio), propesor sa PCC at buhay na "imahe" ng sosyalismo sa Pilipinas. Aktibista para sa wikang Pilipino, mananaysay, at propesor naman sa University of the East si Dizon, kasama siya sa mga tagapagtatag ng KADIPAN.

\section{SI SONNY, BAHAGI NA NG PANITIKANG PILIPINO}

Kilala lamang si Sonny sa pangalan ng kabataang mga manunulat sa MLQ University na nagpangkat sa Mga Agos sa Disyerto, pamagat ng apat-na-edisyong antolohiya $(1964,1974,1993$, at 2010 ng mga premyadong maiikling kathang Pilipino nina Rogelio R. Sicat, Rogelio L. Ordoñez, Dominador B. Mirasol, Edgardo Reyes at ng sumulat nito). Binabasa-basa na namin noon ang mga kritika niya sa panitikan (sa "mahirap intindihing gamit niya ng Ingles"). Kahit hindi nga namin nakakatagpo, parang personal na kakilala namin siya dahil "kaibigan" siya ng mga iniidolo namin - una si Alejandro Abadilla (AGA) na tinutulungan niyang maging "pampanitikan" ang nilalaman ng ineedit nitong Panitikan (revival ng tagapamansag ng Kapisanang Panitikan ng mga manunulat sa Tagalog bago nagkadigma) at si AVH, matapang na manunulat sa pagpaksa ng itinuturing naming "taboo" kung kami ang susulat dahil hindi naman ilalathala ng karaniwang "outlet" naming mga babasahin kundi tanging ang diyaryong Ang Masa na ineedit niya at ibinebenta ng/ 
para sa mga aktibista. Tinitingnan namin si Sonny na "kaisa" ng mga manunulat sa Pilipino, tulad din ni Federico Licsi Espino, Jr. (makata sa Ingles, Pilipino, at Ilocano) na lubhang "malapit" sa matandang makata. Pagkaraang makapaglathala siya ng ilang tula't kuwento sa Panitikan, Liwayway, at Free Press sa Wikang Pilipino hindi namin siya itinuring na "nangangapitbahay" lamang sa aming bakuran. Namangha rin kami sa serye ng mga sanaysay niya sa Panitikan ukol sa pagpapahalaga sa mga "haligi" ng panulaang Tagalog (karamihan ay mga makatang kilala na bago pa nagdigma), sa pormalistang dulog niya sa ebalwasyon ng mga akdang hinanayan ng mga dayuhang teorista/kritiko sa panitikan.

Nang panahong iyon, nailathala na naming "magkabarkada" sa MLQ University ang Mga Agos sa Disyerto (1964) bilang pagtalimuwang sa tuligsa na ang Panitikang Pilipino ay "disyerto," hango na rin sa depensa sa wika't panitikang Pilipino ni Pablo N. Glorioso, isang manunulat na opisyal noon ng Surian ng Wikang Pambansa (KWF ngayon). Inihain namin ang mga katha sa antolohiya bilang "hindi pa nahuhukay na ginto," na kinilala naman ni Dr. Bienvenido Lumbera na nakahihigit sa ilang aspekto sa panitikang Pilipino sa wikang Ingles. Naunahan pa yata ni Sonny ang mga manunulat sa Pilipino sa pagtugon sa paulit-ulit na batikos nina Edith at Edilberto Tiempo (ng Silliman University) sa tula, kuwento at iba pang mga akda sa Pilipino/ Tagalog na para sa dalawa ay "pulos didaktiko, nangangaral...walang kuwenta at mababa ang istandard." Gamit ang mga pag-aaral na lokal at dayuhan na hinubog ng pormalistang "kalakaran" sa edukasyon sa kritisismo (hango ni Sonny sa U.P. at pinalawak pa sa Harvard), umangkop iyon para sa "pakay ko (na) ipagtanggol at patunayan na may angking kalidad" ang Panitikang Pilipino. Kung maagang nabasa namin ang "pagtatanggol" niyang iyon, marahil ay nasulatan namin siya.

Nagkataong nang tinutulungan ni Sonny si AGA, mainit na hindi lamang sa magasing Panitikan kundi pati sa mga pahayagang pangkolehiyo tulad ng Dawn (UE), The Quezonian (MLQU), The Varsitarian (UST) at The Torch (PNC/U), ang polemika ng matatandang makata sa panig ni Ruben Vega at ng kabataang mga makata sa panig naman ni Pedro L. Ricarte. Sa pamamagitan ni Sonny, nagkaroon ng masusi at obhektibo na pagtalakay sa obra ng pangunahing mga makatang sina Teo S. Baylen, Manuel Principe Bautista, Manuel Car. Santiago, Gonzalo Flores, at Federico Licsi Espino, Jr. na nagbigay ng malaking tiwala, ngunit maling nosyon na makabuluhan sa "sukatang Kanluranin" ang sining ng mga akda sa Wikang Pilipino, gayong sa totoo, tiwalag at walang kabuluhan pa rin ang mga tulang iyon para sa masang bumubuo ng kalakhan ng sambayanan. Sa tingin ko, ang mga sanaysay sa kritika ni Sonny ang nagdugtong ng buhay sa magasing Panitikan hanggang matigil ang lathala nito sa bungad ng 1970. Noon pang panahong iyon, kinokondena na ang panitikang "hindi nakikisangkot" sa mga pagtalakay sa mga isyung nakapagmumulat sa masa at mga kilusang pandaigdigan para sa liberasyon ng sambayanan mula sa kolonyal at imperyal na paniniil at pagsasamantala. Dalawang taon pagkaraan 
ng Unang Sigwa (Enero, 1970), kinilala si Sonny ng Surian ng Wikang Pambansa dahil sa aktibong pagsusulat niya sa Wikang Pilipino bilang intelektuwal ng bayan at tagapagpalinaw ng mga isyung pandaigdigan-- nang "buong tapang"siyang hinayaan ng ahensiyang ito ng gobyerno na basahin niya sa Panayam Balagtas'72 ang "Sining at Panitikan sa Rebolusyong Kultural," hatid ang sagot sa mga tanong na: Para Kanino ang Sining at Panitikan? Sino ang Pinaglilingkuran Nito? Ano ang Dapat Gawin? Kung nais mong maging makatotohanan, Kasamang Manlilikha, at maging matapat sa realidad na siyang nagpapagalaw sa kapaligiran, kailangang lumahok ka sa rebolusyonaryong pagsisikap ng masa upang sa gayo'y masakyan mo ang mga kahiwagaan ng karanasan sa buhay at mapanghawakan mo ang mga batas ng kaisipa't damdamin ng tao kaakibat ng mga pagbabagong nag-iiba sa hugis at anyo ng namamalayang daigdig. Winakasan niya iyon sa mataginting na tinig ng: MABUHAY ANG WIKANG PILIPINONG SANDATA NG REBOLUSYON TUNGO SA PAMBANSANG DEMOKRASYA. Ilang buwan pagkaraan, idineklara na ni Marcos ang batas-militar!

\section{BAGONG SIPAT SA SINING NG TULA NI AGA}

Si Sonny ang nagbigay ng introduksiyon sa antolohiyang Mga Piniling Tula ni Alejandro Abadilla (1964). Tulad ng nasabi na, pormalistang dulog ang ginamit niya sa pagpapahalaga sa mga tulang kalakip doon. Pagpapalawak, kung gayon, ang ebalwasyon niya sa mga tula ng pangunahing mga makata ng Wikang Pilipino na inilathala ng magasing Panitikan. Kaya kung iyon na ang huling aplikasyon per se ng pormalismong dulog ni Sonny sa panitikan/lipunan, naghahanda na rin siya sa paggamit ng "bagong lente" sa kaniyang mga pagsusuri sa Panitikang Pilipino. Pagkaraan ng mga pagtimbang niya sa kabuluhan ng mga obra nina Balagtas, Rizal, de Jesus (Jose Corazon), at iba pa, binalingan niya kamakailan ang pagtasa sa sining ni Alejandro Garcia Abadilla.

Maraming bansag na iniuukol kay Abadilla: Ama ng Modernistang Tulang Tagalog, indibiduwalista/egoista, avant-garde; ngunit sa palagay ni Sonny, hindi ito naisakonteksto sa kasaysayan/kamalayan ng panitikan ng bansa. Ikinredito rin noon sa kontrobersiyal na makatang ito ang simula ng gamit ng vers libre gayong nangauna na sina Benigno Ramos, Pedro Gatmaitan, at maging ang ilang makata sa Ingles. Maging ang nagmasteral sa makabagong tula na si Pedro L. Ricarte na buong linaw na nagbigay-pahalaga kay AGA, gamit ang mga pananaw na pampanitikan nina Jean Jacques Rousseau, T.S. Eliot, Leo Tolstoy, Allen Tate, at I.A. Richards ay sinabihan ni Sonny na nagmalabis din sa proklamasyong mapanghimagsik na makata si AGA nang hindi nito (Ricarte) nauugat sa agham-politika at aghamsosyedad ang pagsusuri sa pinagmulan ng tendensiya ng matandang makata. 
Binalikan ni Sonny ang kasaysayan ng mga kilusang pang-ideyolohiya at pampulitika ng ating bansa at doon niya ibinatay ang pagtubo at paglago ng estetika ni AGA na inakala ng iba na senyas lamang ng "ego" nito. Sa sipat ni Sonny, ang buong buhay ni AGA ay saklaw ng panahon ng matinding mga kontradiksiyon sa buhay-Pilipino. Pinanulayan (sa simula) ang pag-iral ng makatang ito ng kaisipang liberalismo na lumaganap at nauwi sa hangad na pantay na mga karapatan ng mga tao at pag-iral ng katarungan para sa lahat mula sa Kilusang Propaganda hanggang panahon ng Rebolusyon, saka nagtuloy hanggang sa brutal na pagsupil ng mga Amerikano sa "insureksiyon" ng mga Pilipino. Naging maikli ang "umano" ay mapagsakripisyong papel ng mga ilustrado sa deklarasyon ng kasarinlan sa Kawit at proklamasyon ng "republika" sa Malolos nang maghukay na si Luna ng mga trinsera sa Kalookan at nagtalaga na ng mga kawal hanggang sa Pampanga. Nagbulag-bulagan ang mga hunyangong ito sa pakunsuwelong alok na asimilasyon ng mga Amerikano na tuluyang nagtaboy kay Aguinaldo sa Palanan. "Naglagalag" si Abadilla at humantong sa Seattle, Washington noong 1929, nag-editor ng pitakTagalog ng Philippine American Review at ng Philippine Digest, bumalik at humingi ng tawad sa ama, nag-aral uli (ng pilosopiya) sa Unibersidad ng Sto. Tomas at nahalal na konsehal sa kanilang bayan ng Salinas (Rosario), Cavite. Noon, wala na ngang maririnig na pagsabog ng mga punglo sa mga nayon at sityo ng kapuluan, nagsasanay na ang mga tinuruan ng "American way" para sa pangakong pagsasarili pagkaraan ng pamahalaang Komonwelt. Bagama't may mga kabalisahan sa mga probinsiya (nag-alsa ang mga Kolorum, Sakdal, at iba pa), nandalang na rin ang malawakan at mainitang pakikipagtagis ng mga unyon sa mga kapitalista, mga magbubukid na kumukuwestiyon sa pakikipaghatian sa mga maylupa, at pati mga babasahin na "palaban" noong unang dekada rito ng mga Puti ay kumitid na ang "espasyo" para sa mga protesta pagdating na ng dekada trenta. Samakatuwid, sa pagkakilala ni Sonny kay AGA sa konteksto ng ganiyang proseso ng kasaysayan, natunton niya ang pagsibol at pagyabong ng pananaw/isip sa estetika ng matandang makata, kaya naging konklusyon niya na ang pagkukunwari, panlilinlang, at pagbabalatkayo sa maraming pagkakataon sa kapaligiran nito ang pinagmumulan ng bulalas ng mga reklamo at "panleletse" nito na repleksiyon ng mga hinagpis ng masa nang panahong iyon, ayon kay Sonny. Hindi rin propeta ng sambayanang Pilipino na "tagapagpahayag ng paniwala at damdamin at karanasan" si Abadilla, gaya ng sinabi ni Ricarte, salungat sa opinyon ni Abueg na hindi ang "sentimental na pagpukaw sa damdamin ang layunin ni AGA kundi ang pagdidili-diling hubad sa emosyon o pangangaral." Matapat ngang makata si AGA, ngunit biktima siya ng masalimuot na buhay ng lipunang kaniyang iniralan na naglimita sa kapasidad niya bilang Manlilikha. Katulad ni AVH at iba pang kalinya nito ang kaisipan at hangarin, si Abadilla ay alaalang muhon sa ating panitikan. 


\section{E. SAN JUAN, JR., ISA PANG MAPAGPALAYANG PANULAT}

Malaki na ang corpus ng mga nasulat ni Sonny sa wikang Filipino (tawag na rito batay sa Konstitusyon ng Pilipinas, 1986), mula sa kaniyang mga "pagsasanay" na nalathala sa mga pahayagang pang-estudyante sa kolehiyo at mga unibersidad. May mga tula siya sa Philippine Collegian, Dawn, at mga kuwento sa Panitikan, Liwayway, at Free Press sa Wikang Pilipino saka pagsusuri sa Katas, Taliba, at Dimension (UE). May mga antolohiya siya ng mga kuwento, Ang Mangwawasak at iba pang Katha, at ng maraming mga tula mula Maliwalu (1970) hanggang Kundiman sa Gitna ng Karimlan, nagwagi ng National Book Awards (2014), tipon niya sa tatlong dekada ng pagsulat niya ng tula sa Pilipino. Sa pagsusuri, nagsimula siyang maglathala ng aklat tungkol sa mga paksang Pilipino sa Rice Grains: Selected Poems ni Amado V. Hernandez (1966), Ang Sining ng Tula (1965) at iba pa hanggang sa Lupang Hinirang, Lupang Tinubuan: Mga Sanaysay sa Kritika, Kasaysayan, at Politikang Pangkultura (2015). Iisa ang nangingibabaw na layunin niya sa pagsulat: pagpapamulat at pagkilos tungo sa pagtatamo ng kinabukasang ibig ni AVH na mangibabaw sa Pilipinas: demokrasya na di lamang karapatan ng nakararami ang mamahala kundi kapantay na karapatan din ng kakaunti ang sumalungat.

Masasabing pasalunga sa agos si Sonny hinggil sa usapin sa gagamiting wika sa pagsulat. Kung ang seminal na tagumpay ni Paz Marquez sa pagsulat sa panitikan natin sa wikang Ingles ay pinagsumundan ng kabataang mga manunulat, sa sigasig ngayon ni Sonny sa pagsulat sa wikang Filipino makit niya ang kabataang mga manunulat na makisangkot sa ipinaglalaban niya. Habang naglulunoy pa rin ang maraming manunulat natin sa mga teoryang pangkaisipan at pangsikolohiya, abstrakto at obskyurantiko, lalong nagpapalalim si Sonny ng pag-aaral sa estetiko at panitikang hindi maihihiwalay sa buhay ng lipunan at nakapagpapalinaw sa mga kontradiksiyon nito. Mula sa pagtalikod sa mga uri ng panitikang natutuhan sa U.P. at sa Harvard, ganap na saligan niya at nililinang pa ang diyalektiko-materyalismong analisis ng sining at lipunan, katuwang lamang sa paglilinaw ang mga teoryang formalismo, istruktural, at iba pang kalakarang hindi sumusulong, kundi nababaon pa nang malalim sa kinasadlakang mga hukay. Kaya mula sa pagkritika ng mga pundasyon ng panitikan ng ating bansa hanggang sa ebalwasyon ng kasalukuyang mga manunulat na sumasabay sa pagpapaliwanag ng hegemonya ng yaman at kapangyarihang global na patuloy na umiiral ngayon, ang mga akda niya sa sarili't natutuhan niyang wika ang magpapalaya sa mga kaisipang kadlo ng hawla ng mga puwersang ganid hangga't nakapapangibabaw sa isip at sikmura ng sangkatauhan. 